\title{
Success Rates Depend on the Appropriateness of the Outcome Measures
}

\author{
Kenneth Royal \\ North Carolina State University
}

This "Cheap Lessons" article emphasizes the importance of selecting an appropriate outcome. A brief example from the sports medicine literature relating to baseball pitching performance and the "Tommy John surgery" (TJS) is discussed. The example illustrates how an inappropriate outcome measure often utilized in studies involving the TJS has resulted in deceptive, overestimates of the surgery's success rate. The unintended consequence of this methodological miscalculation is that numerous athletes have elected to pursue this unnecessary medical procedure and put both their health and sporting careers at risk.

Keywords: Methodology; Research Design; Measurement; Statistics; Data Analysis; Outcomes Assessment

A common practice in health outcomes assessment is to determine the success rate of a treatment. However, determining an appropriate outcome is no trivial matter. An article from Yurkiewicz (2015) on sports medicine and baseball pitchers provides an excellent example.

A popular surgical procedure called ulnar collateral ligament (UCL) reconstruction was made famous in 1974 when Tommy John, a major league baseball pitcher, underwent the surgery. The procedure is known by many as the "Tommy John surgery" (TJS), and involves replacing the UCL in the medial elbow with a tendon from elsewhere in the body (often from the forearm, opposite elbow or patella). The procedure is widely recognized for having a success rate of around 90-93\%. Because of such reportedly high success rates, many high school athletes, parents, collegiate athletes, and coaches believe the surgery should be performed on players without elbow injuries as a means to enhance performance (Ahmad, Grantham, \& Greiwe, 2012).

Success rates for many medical procedures, including UCL surgeries, often are measured by way of a rehabilitation questionnaire in which persons who recently completed a procedure answer numerous survey items about perceived strength, comfort, sleep quality, ability to complete a variety of daily activities, and so on. While patient surveys generally are helpful for discerning a patient's path to recovery, these surveys often provide insufficient and inappropriate outcome measures. This recently was evidenced with the case of UCL surgeries. More specifically, when sports medicine physicians began comparing pre- and post-UCL surgery performance for each individual (e.g., using each subject as his/her own control) they found that only $70-75 \%$ of pitchers were able to replicate the 
same types of baseline performance after the surgery (Yurkiewicz, 2015). The lesson here was that when the outcome variable was changed, so did the success rate.

As outcomes assessment becomes increasingly personalized in medicine via the 'precision medicine' movement (Chouchane, 2011; Topol, 2014), there likely will be an increased number of studies that focus on person-level performance with each person serving as his or her own control. Ideally, these studies will utilize multiple measurements at several time points to both better understand any resulting changes and control for regression to the mean. While studies focusing on personalized outcomes is perhaps a methodological step forward, the fact remains that researchers need to make every effort to get as close to the appropriate outcome measure as possible. Thus, while patient surveys typically offer a convenient and relatively inexpensive way to collect data and assess patient outcomes, failing to identify the best outcome measure and subsequently evaluating it may result in serious, negative unintended consequences for patients.

In the case of UCL surgeries, many members of the general public remain highly influenced by purported success rates. Thus, some individuals will be more likely to undergo this unnecessary medical procedure because of its supposed effectiveness. Failing to properly identify an appropriate outcome measure can not only threaten the quality of a research study, but also put patients' health at risk. As responsible researchers and methodologists, we need to remain extremely cognizant of the importance of selecting appropriate outcome measures.

Author Notes: Kenneth Royal is Assistant Professor of Educational Assessment \& Outcomes, North Carolina State University, College of Veterinary Medicine, Department of Clinical Sciences, 1060 William Moore Dr., Raleigh, NC 27609 USA. Email: kdroyal2@ncsu.edu.

\section{References}

Ahmad, C. S., Grantham, W. J., \& Greiwe, R. M. (2012). Public perceptions of Tommy John surgery. The Physician and Sports Medicine, 40, 64-72.

Chouchane, L., Mamtani, R., Dallol, A., \& Sheikh, J. (2011). Personalized Medicine: A Patient-Centered Paradigm. Journal of Translational Medicine, 9, 1-3.

Topol, E. J. (2014). Individualized Medicine from Prewomb to Tomb. Cell, 157, 241-253.

Yurkiewicz, S. (2015). Did UCL Surgery Work? Baseball Stats May Have the Answer. Medpage Today. Available at:

http://www.medpagetoday.com/SportsMedicine/EliteSports/51184. 